\title{
Variabilidad de la precipitación y su relación con los rendimientos agrícolas en una región semiárida de la llanura pampeana (Argentina)
}

\section{Rainfall variability and its relationship with the agricultural yields in a semiarid region of the Pampean plain (Argentina)}

\author{
Andrea Soledad Brendel ${ }^{1}$, Vanesa Yael Bohn² y María Cintia Piccolo ${ }^{3}$
}

\section{RESUMEN}

El objetivo de este estudio fue analizar la alternancia de períodos secos, húmedos y normales durante los años 1970-2012 en una región semiárida de la llanura pampeana argentina. La evolución de los rendimientos de un cultivo de secano (trigo) se analizó como indicador de dicha alternancia, durante el período 2000-2012. Se utilizó el Índice Estandarizado de Precipitación y Evapotranspiración (en inglés SPEI) y se analizó la superficie sembrada, cosechada y los rendimientos del cultivo de trigo. Los períodos normales según el SPEI fueron los más frecuentes en el área de estudio mientras que hubo una desigual proporción en la ocurrencia de eventos se-

${ }^{1}$ Instituto Argentino de Oceanografía (IADO - CONICET) y Universidad Nacional del Sur, Departamento de Geografía y Turismo y Departamento de Agronomía, Argentina. andreabrendeluns@gmail.com o asbrendel@iado-conicet.gob.ar ORCID iD: http://orcid.org/0000-00020909-4694.

${ }^{2}$ Universidad Nacional del Sur, Departamento de Geografía y Turismo y Consejo Nacional de Investigaciones Científicas y Técnicas (CONICET), Argentina. vbohn@criba.edu.ar ORCID iD: http://orcid.org/0000-0002-4497-7556.

${ }^{3}$ Instituto Argentino de Oceanografía (IADO - CONICET) y Universidad Nacional del Sur, Departamento de Geografía y Turismo, Argentina. mcpiccol@gmail.com ORCID iD: http://or cid.org/0000-0002-5184-9149. 
cos y húmedos. El año 2001 fue húmedo y los años 2008 y 2009 fueron secos. Durante estos últimos, disminuyeron los rendimientos y la extensión de la superficie cosechada de trigo, mostrándose como indicadores de la alternancia de períodos secos y húmedos.

PAlabras ClaVE: variabilidad de la precipitación; región semiárida; Índice Estandarizado de Precipitación y Evapotranspiración; cultivo de trigo; períodos secos y húmedos.

\section{AbSTRACT}

The aim of this work was to analyze the dry, humid and normal periods during the 1970-2012, in a semiarid region of the Pampean plain (Argentina). In addition, the evolution of yields of rainfed crop (wheat) was evaluated during the 2000-2012 years. The Standardized Precipitation and Evapotranspiration Index (in English, SPEI) was applied and the wheat crop evolution was analyzed. Normal periods and dry and humid periods showed a high a low frequency in the study area, respectively. The longest wet periods occurred during the 1970-1980 years. The 2001 was categorized as a wet year. The 2008 and 2009 were dry. Wheat crop evolution during the study period was shown as an indicator of the dry and wet periods alternation.

KEY WORDS: rainfall variability; semiarid region; Standardized Precipitation Evapotranspiration Index; wheat crop; wet and dry periods.

Cómo citar este artículo / Citation: Brendel, Andrea Soledad, Bohn, Vanesa Yael y Piccolo, María Cintia (2017): "Variabilidad de la precipitación y su relación con los rendimientos agrícolas en una región semiárida de la llanura pampeana (Argentina)", Estudios Geográficos, LXXVIII/282, pp. 7-29.

\section{INTRODUCCIÓN}

La variabilidad climática depende de numerosos factores, dado que es producto de eventos meteorológicos que acontecen a diferentes escalas espaciales y temporales (décadas, años, estaciones). Los períodos secos y húmedos son un aspecto de la variabilidad climática y constituyen uno de los fenómenos climáticos de mayor impacto en la hidrología y en la actividad agrícola de la región pampeana argentina (Bohn et al., 2011). En un período relativamente extenso, la sequía corresponde a intervalos de déficit pluviométrico respecto del valor normal. La ocurrencia de una sequía depende de la evapotranspiración, del déficit de las precipitaciones y del condicionamiento impuesto por los cambios en la presión atmosférica causantes de las alteraciones en la circulación general de la atmósfera (Piccolo et al., 2002). 
La precipitación es uno de los elementos climáticos de mayor importancia para el hombre debido a que no sólo influye en la configuración del medio natural sino que también condiciona las actividades humanas. Es importante conocer su distribución y comportamiento porque interviene directamente en la economía de una región, sobre todo en aquellas donde las precipitaciones son escasas (Gómez y Pérez, 2011). Las regiones semiáridas presentan gran vulnerabilidad a la sequía debido a las escasas precipitaciones y a su variabilidad espacial y temporal (Askew y Rodda, 1996). En la región semiárida del centro y norte de Chile, las sequías son eventos recurrentes de gran impacto socioeconómico (Fernández y Vergara, 1998). En la cuenca de Guadalentín, ubicada al sureste de España, la disminución de las precipitaciones primaverales y otoñales afectan los principales cultivos de secano, especialmente el trigo y la cebada (García Marín, 2006). Durante el período 1990-1994, en ese país, se registraron importantes descensos de los rendimientos del cultivo de trigo debido a la sequía (López Bermúdez y Sánchez Fuster, 1997). Por su parte, en una región semiárida ubicada al noreste de Irán, la sequía es una de las principales causas de la variabilidad del rendimiento interanual de trigo (Bannayana et al., 2010). En Australia, como consecuencia de la sequía ocurrida durante los años 2002 y 2003, la producción agrícola se redujo un 30\% (Horridge et al., 2005).

Las fluctuaciones de algunos parámetros del clima inciden en la rentabilidad agropecuaria a través de su impacto directo sobre el avance de los cultivos y los rendimientos, los usos del suelo, entre otros (Campo et al., 2012). Numerosos estudios coinciden en que las precipitaciones y su variabilidad constituyen el factor que genera más incertidumbre en la producción agropecuaria (Sebalt, 2002). Estudios realizados en la región pampeana argentina señalan que en los años «Niño» las precipitaciones tienden a ser superiores a lo normal, especialmente entre los meses de noviembre a enero, mientras que en los años «Niña» las lluvias tienden a ser inferiores a lo normal entre octubre y diciembre. (Grondona, 2007). Estas variaciones cíclicas influyen en los rendimientos de los principales cultivos de la zona pampeana: trigo, maíz, girasol y soja (Grondona, 2007).

El área de estudio, la porción bonaerense de la región semiárida pampeana (RESAB), se caracteriza por la alternancia de períodos secos y húmedos y la producción de cultivos de secano. La precipitación constituye la principal entrada de agua dentro del sistema de producción realizado en la región ejerciendo un importante control sobre la productividad de los cultivos invernales. Las variaciones del clima y principalmente de las precipitaciones constituyen el factor más importante en la variabilidad de los resultados de los 
sistemas de producción agrícolas argentinos (Hall et al., 1992). Según Hurtado y Berri (2001), en la región pampeana, los rendimientos del cultivo de trigo se ven influenciados por las variaciones periódicas y cuasicíclicas de las condiciones climáticas.

Debido a que la magnitud y duración de estos períodos impactan en el desarrollo de las actividades de la sociedad, es imprescindible un estudio minucioso del fenómeno. El Índice Estandarizado de Precipitación y Evapotranspiración (SPEI) se presenta como una herramienta eficiente para su análisis. El objetivo de la presente investigación es analizar la alternancia de períodos secos, húmedos y normales en la porción bonaerense de la región semiárida pampeana durante el período 1970-2012. Complementariamente, se evaluará la evolución de los rendimientos de un cultivo de secano (trigo) como indicador de dicha alternancia durante un período menor (2000-2012).

\section{ÁREA DE ESTUDIO}

El área de estudio se denomina Región Semiárida Bonaerense (RESAB) y forma parte de la llanura pampeana argentina. La misma incluye las ecoregiones Pampas y Espinal (Viglizzo et al., 2011) extendiéndose entre los $30^{\circ}$ y $38{ }^{\circ} \mathrm{S}$ (Iriondo, 1989). Esta región también fue denominada planicie central argentina (Diovisalvi et al., 2014). Es una extensa planicie de aproximadamente 50 millones de hectáreas (Álvarez y Lavado, 1998) y es considerada una de las regiones agrícolas más productivas del mundo (Satorre y Slafer, 1999). Desde el punto de vista geomorfológico, el territorio objeto de estudio tiene una suave inclinación norte-sur desde Sierra de la Ventana hasta la línea de costa (figura 1). Está formada por sedimentos no consolidados de edad cuaternaria, aportados en su mayor parte por el viento y redistribuidos por acción hídrica.

El clima es frío subhúmedo o semiárido (Díaz y Mormeneo, 2002) con máximas precipitaciones en primavera y otoño. Éstas, al igual que la temperatura presentan variaciones espaciales en relación con la continentalidad, paso de sistemas frontales, orientación de la costa y corrientes oceánicas. Las temperaturas medias anuales están comprendidas entre $14^{\circ} \mathrm{C}$ y $20^{\circ} \mathrm{C}$. Las precipitaciones no siempre suficientes en los sectores occidentales para el cultivo sin riego otorgan un carácter subhúmedo a esta variedad de clima templado, denominado también de transición (Campo et al., 2004). Los suelos de la región son aptos para la agricultura y la ganadería, dicha aptitud decrece en relación al gradiente de isoyetas anuales (de noreste a sudeste) (Brown et al., 2006). 
FIGURA 1

LOCALIZACIÓN DEL ÁREA DE ESTUDIO

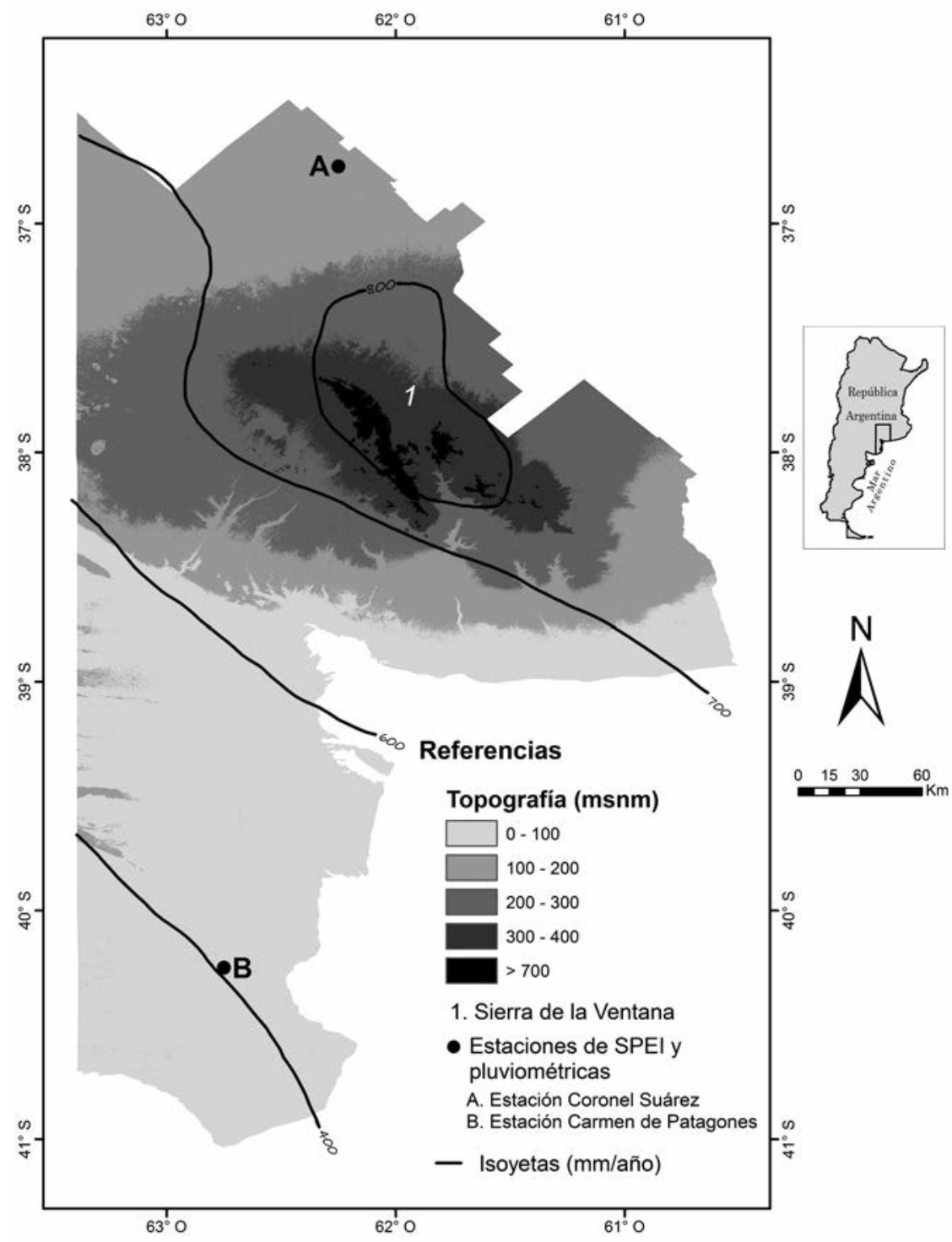

Fuente: elaborado sobre la base del ASTER GDEM y AEROTERRA. 
El trigo es el principal cultivo de cosecha y es la base de los sistemas productivos del área de estudio. Aporta el 12\% del total de la producción nacional (Coma, 2010). El cultivo de trigo en la región representó mucho más que un fenómeno agronómico y económico, fue el fundador de la agricultura extensiva del área de estudio. Su desarrollo no solamente aportó beneficios económicos sino que también cambió radicalmente la estructura social y cultural de los pueblos de la región (Ponzio et al., 2013).

A partir de 1970, la superficie sembrada del cultivo de trigo en el área de estudio mostró un constante decrecimiento. Este aspecto pudo estar incentivado por las condiciones climáticas adversas y por las expectativas zonales puestas en otro tipo de cultivo de cosecha (oleaginosas), retenciones a las exportaciones y bajos precios del cereal (Coma, 2010). A pesar de esto, el trigo constituye la base económica de la región.

\section{MATERIALES Y MÉTODOS}

El estudio de la variabilidad de la precipitación incluyó el análisis de registros de precipitación in situ así como también el de registros del SPEI (Vicente-Serrano et al., 2010). En el primero de los casos, se analizaron datos pluviométricos anuales (período 2000-2012) correspondientes a las bases de datos del Servicio Meteorológico Nacional (SMN) y el Instituto Nacional de Tecnología Agropecuaria (INTA). La región fue analizada en 2 subzonas, delimitadas según la cantidad anual de precipitación: el sector norte, entre la isoyeta de 800 y 700 mm/año (estación Coronel Suárez) y el sector sur, entre la isoyeta de 600 y $400 \mathrm{~mm} /$ año (estación Carmen de Patagones) (figura 1). Para la identificación y análisis de períodos secos, húmedos y normales y su variación espacial y temporal se aplicó el SPEI.

La serie de datos fueron obtenidos de la página Web del SPEI, (http://sac. csic.es/spei/home.html) durante el periodo 1970-2012, a una escala espacial de $0,5^{\circ}$. El SPEI es un índice climático mensual calculado a partir de la diferencia entre la precipitación acumulada en un período de tiempo y la evapotranspiración potencial. La utilización de este último parámetro es la diferencia más importante con respecto al Índice Estandarizado de Precipitación (SPI), porque permite detectar, analizar y monitorear la sequía de manera más acertada, de acuerdo a valores de referencia (tabla 1). Además, puede ser calculado para diferentes escalas de tiempo (desde 1 a 48 meses). 
TABLA 1

VALORES DEL SPEI

\begin{tabular}{|c|c|}
\hline SPEI & CATEGORÍAS \\
\hline 2,0 o más & Extremadamente húmedo \\
1,5 a 1,99 & Muy húmedo \\
1,0 a 1,49 & Moderadamente húmedo \\
$-0,99$ a 0,99 & Casi normal \\
$-1,0$ a $-1,49$ & Moderadamente seco \\
$-1,5$ a $-1,99$ & Muy seco \\
$-2,0$ o menos & Extremadamente seco \\
\hline
\end{tabular}

Fuente: Vicente Serrano et al. (2010).

Según Mckee et al. (1995) las condiciones de humedad en el suelo responden a anomalías de precipitación para una escala temporal relativamente corta de 1,3 y 6 meses, mientras que el agua de las corrientes superficiales, subterránea y reservorios reflejan anomalías de lluvia de períodos más largos: escalas temporales de 12, 24 y 48 meses. Por este motivo, se evaluó la escala temporal de 3 meses para el período 1970-2012. Además se graficó la serie 1970-2012 y se analizaron todas las categorías tanto de humedad como de sequía calculando además el porcentaje de cada una de ellas. En relación al estudio de los rendimientos como indicadores de la alternancia de períodos secos, húmedos y normales, se analizaron datos de superficie sembrada (ha) y superficie cosechada (ha) y los rendimientos del cultivo de trigo ( $\mathrm{kg} / \mathrm{ha}$ ), para la zona norte y sur del área de estudio (figura 1) (campañas 2000/2001 a 2012/2013) cedidos por el Ministerio de Agricultura, Ganadería y Pesca de la Nación (MAGyP).

\section{RESULTADOS Y DISCUSIÓN}

Evolución del SPEI (período 1970-2012)

Como resultado del análisis del SPEI (escala 3 meses) se obtuvo la tendencia de la precipitación en el área de estudio. El sector norte (figura 2) reflejó alternancia de períodos secos y húmedos. Los períodos húmedos representa- 
ron el 20\% de los valores históricos mientras que el 17\% de los valores correspondieron a eventos secos. Los períodos normales representaron el 63\% de los registros. Dentro de las condiciones de humedad, la situación de moderada humedad representó el 13\%. El 5\% y 2\% pertenecieron a las categorías «muy húmedas» $\mathrm{y}$ «extremadamente húmedas» respectivamente. El mayor porcentaje en relación a la sequía correspondió a la categoría de «moderadamente seco» (9\%) mientras que los porcentajes restantes correspondieron a las situaciones de «muy seco» (7\%) y «extremadamente seco» (1\%). Las condiciones de extrema humedad (SPEI $\geq 2$ ) se observaron en los meses de diciembre de 1976, febrero de 1977, septiembre de 1978, abril-junio de 1980, noviembrediciembre de 1985 y enero de 1986 y marzo de 1992 (figura 3). Por su parte, las condiciones de extrema sequía (SPEI $\leq-2)$ se dieron en los meses de diciembre de 1998, junio de 2005 y enero-febrero de 2009 (figura 4). La cantidad de meses con extrema humedad fueron mayores que los de extrema sequía para esta zona y el máximo valor perteneciente a la categoría «extremadamente húmedo» ocurrió en el mes de noviembre de 1985 con un valor de SPEI de 2,52. El máximo valor de sequía extrema se dió en el mes de diciembre de 1998 con un SPEI de -2,30 (figuras 2 y 3).

Durante los años 1970-1974 predominaron los períodos normales y en lapso 1975-1981 hubo alternancia de eventos secos y húmedos. Dentro de este período, los secos se dieron en mayor extensión durante 1975/76 (septiembre-enero), 1977 (mayo-septiembre) y 1981 (agosto-diciembre); y los húmedos en 1976-1977 (octubre-marzo) y 1980 (abril-junio). En el año 1995, se registraron 5 meses consecutivos con condiciones muy secas (de julio a noviembre) (figura 3). Hasta mediados de 1994 predominaron las situaciones húmedas, siendo los años 1992 y 1993 muy húmedos, y a partir de 1995, el comienzo de un período seco (figuras 2 y 3). Estos resultados coinciden con lo estudiado por Piccolo et al. (2002) en su estudio de la sequía de 1995 en el sur de la región pampeana argentina. El déficit hídrico provocó una reducción del 20\% de la superficie cultivada. Esta sequía estuvo caracterizada por temperaturas normales a frías y por la presencia de dos centros de altura geopotencial anómalos: uno negativo sobre el océano Atlántico y otro positivo sobre el Pacífico sudeste (Rivera, 2014).

Otro de los períodos secos de importante duración y magnitud (8 meses) ocurrió en 1998 y principios de 1999 (figura 2). Estos resultados coinciden con lo hallado por Castellarín et al. (1999) en Oliveros, una localidad ubicada al sureste de la provincia de Santa Fé (Argentina). Estos autores determinaron que durante los meses de agosto y septiembre de 1998, las precipitaciones fueron escasas, generando un importante déficit en el balance hídrico. 
FIGURA 2

SPEI EN EL NORTE DEL ÁREA DE ESTUDIO (ESCALA 3 MESES)

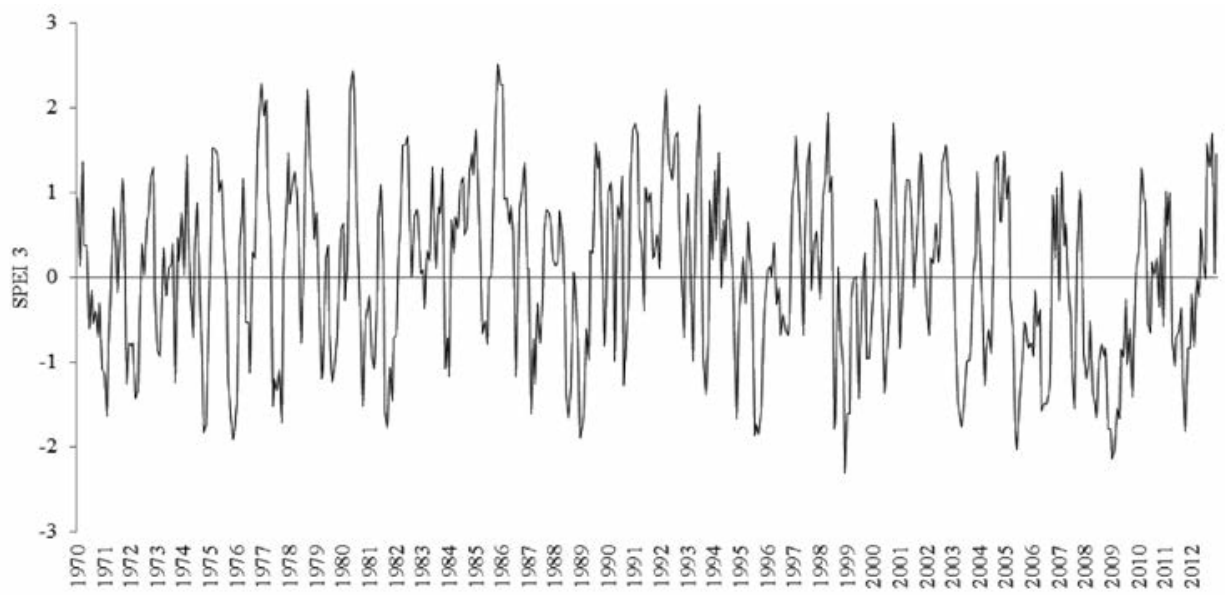

Fuente: elaborado sobre la base de registros de SPEI (http: //sac.csic.es/spei/home.html).

FIGURA 3

VARIACIÓN MENSUAL DEL SPEI DURANTE EL PERÍODO 1985-1995. SECTOR NORTE DEL ÁREA DE ESTUDIO

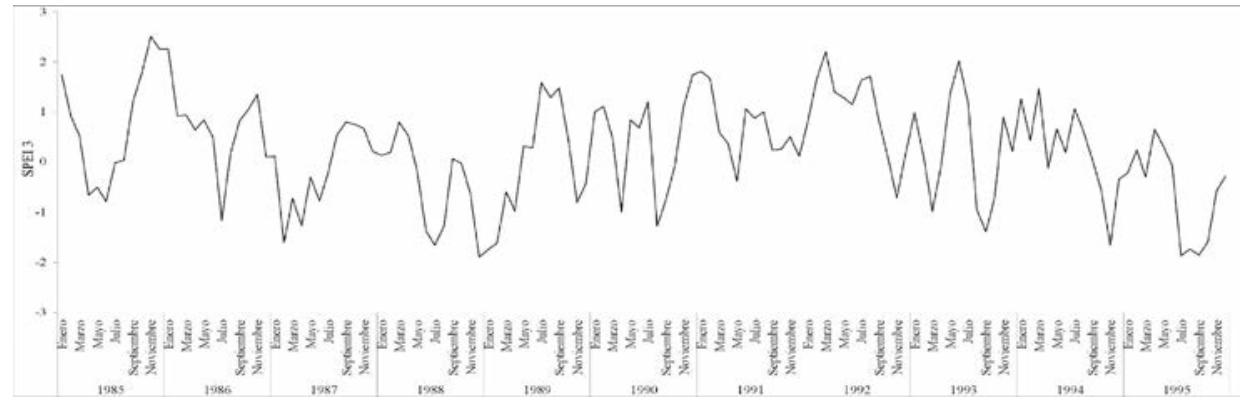

Fuente: elaborado sobre la base de registros de SPEI (http: //sac.csic.es/spei/home.html).

Desde el año 2000, y hasta 2004, predominaron los períodos húmedos. El año 2000 fue normal mientras que el año 2001 presentó condiciones muy húmedas durante varios meses consecutivos (figura 2). Por su parte, el año 2002 tuvo alternancia de condiciones normales, muy húmedas y moderadamente húmedas. Durante el año 2003 ocurrió un período moderadamente seco y 
muy seco desde marzo a junio, siendo normal hasta febrero de 2004. A partir de allí y hasta mediados de 2005, sucedieron condiciones húmedas. El SPEI mostró, a partir de 2005 y hasta 2009, situaciones secas (figura 4). A partir del año 2010 y hasta mediados de 2012, se presentaron condiciones normales seguidas de húmedas que se extendieron hasta finales de 2012 (figura 2). En el área de estudio, el año 2001 fue definido como húmedo mientras que los años 2008 y 2009, como secos encontrando similitudes en lo estudiado para el mismo área por Bohn et al. (2011) y para el oeste del área de estudio por D'Ambrosio et al. (2013).

FIGURA 4

VARIACIÓN MENSUAL DEL SPEI DURANTE EL PERÍODO 2005-2012. SECTOR NORTE DEL ÁREA DE ESTUDIO

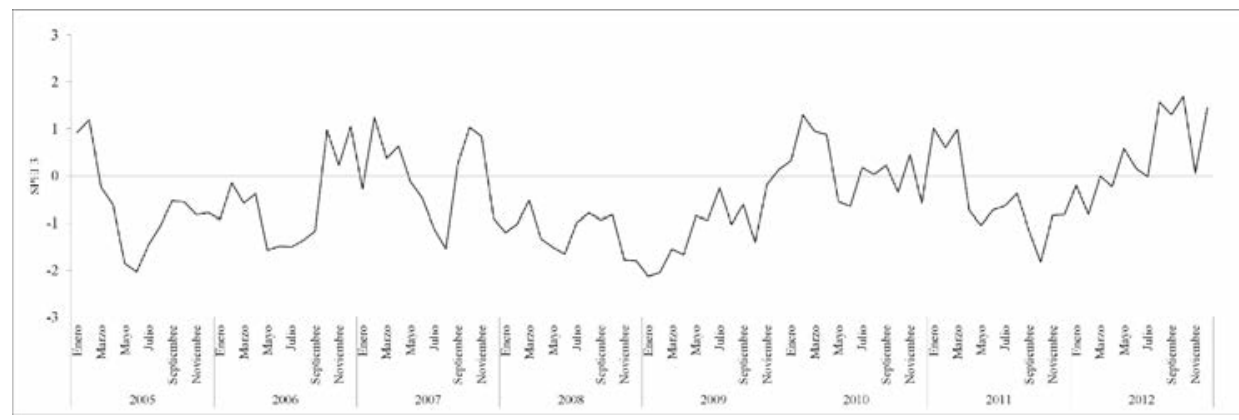

Fuente: elaborado sobre la base de registros de SPEI (http: //sac.csic.es/spei/home.html).

El análisis del SPEI para el sur del área de estudio (figura 5) mostró homogeneidad en la frecuencia de períodos secos y húmedos, representando el 18\% de los valores históricos, respectivamente. Por su parte, el 64\% de los valores históricos correspondieron a eventos normales (ejem.: 1990 y 2000) (figura 8). Los valores extremos de humedad sucedieron en los meses de agosto y septiembre de 1971, diciembre de 1976 y enero y febrero de 1985 y representaron menos del 1\% del total de los datos para el período 1970-2012 mientras que el $11 \%$ y $5 \%$ fueron moderadamente húmedos y muy húmedos, respectivamente. Enero de 1985 fue el mes más húmedo (SPEI: 2,30) (figuras 5 y 6).

Durante 1970-2012, el 11\% correspondió a sequías moderadas, un 6\% a muy secas y sólo $1 \%$ a extremadamente secas (figura 8). Los eventos secos fueron más numerosos que los húmedos y ocurrieron en los meses de diciembre de 1989, julio de 1998 y febrero-junio y agosto-diciembre de 2008, siendo 
extremadamente seco en diciembre de ese año (SPEI: -2,11) (figura 7). En el año 2009, en la mayoría de los meses las situaciones fueron secas con tendencia a la sequía extrema. Se observó que el mes más seco fue junio de 2009 (SPEI: -2,43) (figuras 5 y 7). En Zavalla ( $33^{\circ} 0100$ S; $60^{\circ} 5300$ O), una localidad ubicada al noreste de la llanura pampeana argentina, la sequía ocurrida durante el año 1998 afectó en forma significativa las actividades agropecuarias y en particular los rendimientos del cultivo de trigo (Coronel et al., 2004).

Durante todo el período analizado y al igual que en la zona norte se obtuvo el predominio de condiciones normales. En el período 1970-1975, la tendencia de situaciones húmedas y secas fue similar. A partir de 1976 y hasta 1978 predominaron los períodos húmedos, siendo de mayor magnitud y duración a finales de 1976 y principios de 1977 (figura 5). Los resultados hallados durante la década de 1970, específicamente entre los años 1976 y 1978, coincide con lo analizado por Carbone et al. (2008) en su estudio de períodos secos y húmedos en una cuenca hidrográfica cercana al área de estudio (provincia de Buenos Aires, Argentina) y con lo hallado por Scian (2002) y Bohn et al. (2011) en el área de estudio, haciendo referencia a que durante la década de 1970 toda la región experimentó condiciones hídricas excepcionales.

Entre los años 1979 y 1983 se advirtió la alternancia de períodos secos, húmedos y normales. Los años 1981 y 1982 presentaron 6 meses de condiciones muy secas intercaladas con situaciones normales. A partir de 1983 y hasta 1985 se presentó un período húmedo (figura 6). Desde mediados de 1985 y hasta 1988, las condiciones fueron normales. Durante 1989 y principios de 1990 la situación fue seca siendo extrema en diciembre de 1989 (SPEI= -2,28) (figura 6). Los eventos normales ocurrieron durante 1990 hasta 1996 (con algunos meses de categorías muy secas y moderadamente secas). La distribución del SPEI mostró la presencia de un período húmedo relativamente extenso entre los años 1997 y 1985 (de enero de 1997 a marzo de 1985). El último semestre de 1986 fue seco, siendo extremadamente seco en julio y muy seco en el resto de los meses. Al igual que en la zona norte, el año 2000 fue normal. Se extendió durante el año 2001 un período húmedo, caracterizado por situaciones moderadamente húmedas y muy húmedas en concordancia con lo ocurrido en el año 2004 (figura 5). Por su parte, en el año 2002 las categorías predominantes fueron las normales intercaladas con categorías muy húmedas. La tendencia en el año 2003 fue normal. Los años 2005, 2006 y 2007 presentaron alternancia de eventos secos, húmedos y normales (figura 5).

A partir de 2008 y hasta 2011 se desarrolló un extenso período seco acentuándose en los años 2008 y 2009, definidos como muy seco y extremadamente seco, respectivamente (figura 7). Durante el año 2012 hubo alternancia 
de meses pluviométricamente normales con húmedos y secos (figuras 5 y 7 ). Estos ciclos de déficits y excesos hídricos constituyen uno de los fenómenos climáticos de mayor impacto en la hidrología y en la actividad agrícola de la región pampeana argentina (Bohn et al., 2011).

\section{FIGURA 5}

SPEI EN EL SUR DEL ÁREA DE ESTUDIO (ESCALA 3 MESES)

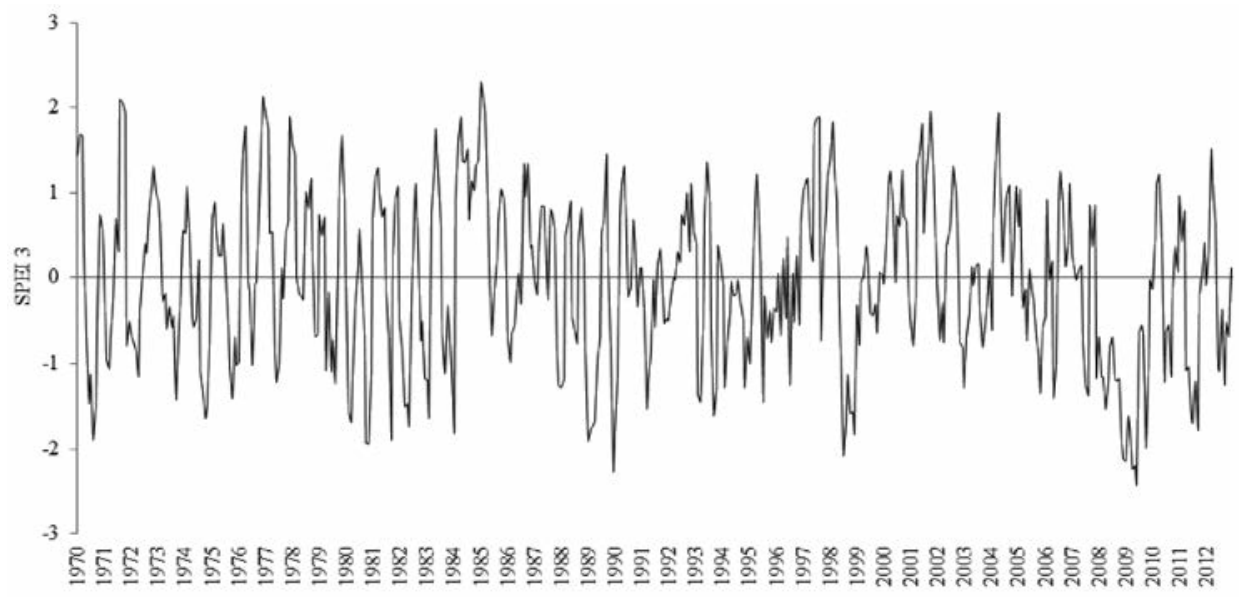

Fuente: elaborado sobre la base de registros de SPEI (http: //sac.csic.es/spei/home.html).

FIGURA 6

VARIACIÓN MENSUAL DEL SPEI DURANTE EL PERÍODO 1983-1990. SECTOR SUR DEL ÁREA DE ESTUDIO

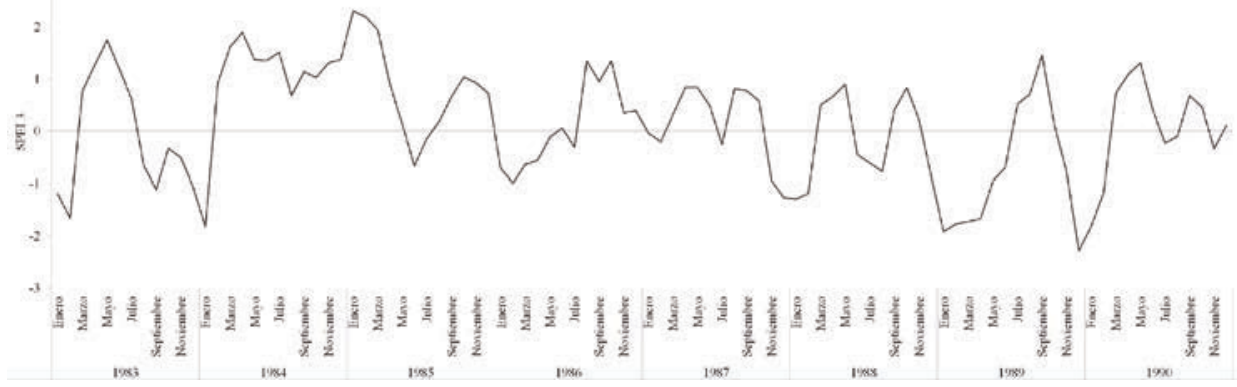

Fuente: elaborados sobre la base de registros de SPEI (http: //sac.csic.es/spei/home.html).

Estudios Geográficos, Vol. LXXVIII, 282, pp. 7-29, enero-junio 2017

ISSN: 0014-1496, eISSN: 1988-8546, doi: 10.3989/estgeogr.201701 
FIGURA 7

VARIACIÓN MENSUAL DEL SPEI DURANTE EL PERÍODO 2008-2012. SECTOR SUR DEL ÁREA DE ESTUDIO

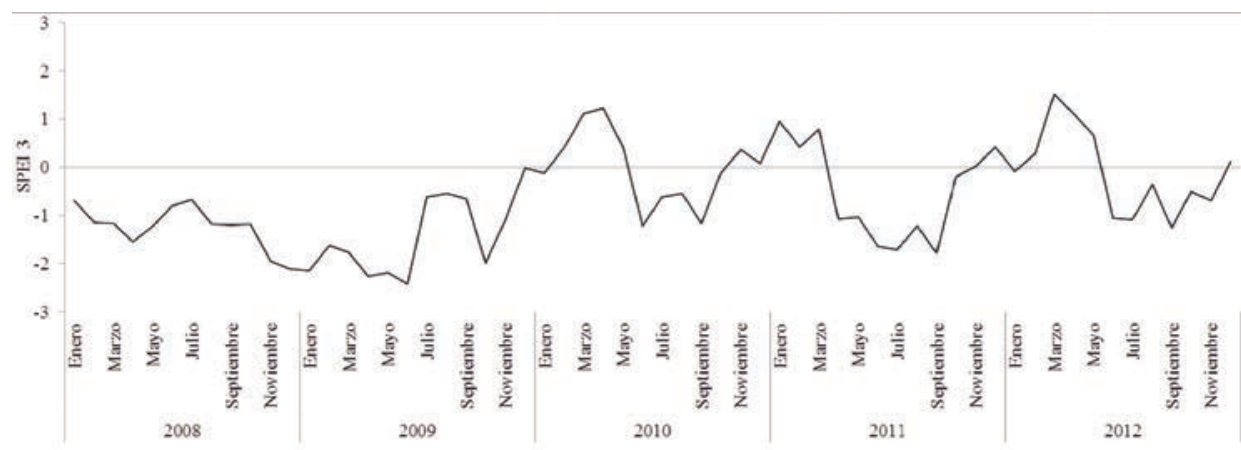

Fuente: elaborado sobre la base de registros de SPEI (http: //sac.csic.es/spei/home.html).

Teniendo en cuenta el período analizado (42 años), el sector sur presentó el $1 \%$ de condiciones de extrema sequía y el $2 \%$ de extrema humedad. Por su parte, las situaciones muy húmedas y muy secas obtuvieron valores del 5\% y $6 \%$ respectivamente, mientras que las categorías moderadamente húmedas y secas alcanzaron un $11 \%$. El porcentaje restante (64\%) correspondió a situaciones normales (figura 8). La situación analizada en el norte del área de estudio, coincide con lo ocurrido en el sector sur. Los valores más bajos correspondieron a las categorías extremadamente seco y húmedo ( 1 y $2 \%$ respectivamente). Se registraron un 5\% de situaciones muy húmedas y un $7 \%$ de muy secas. Los eventos de moderada humedad ocurrieron en un 13\% y los de sequía en un 9\%. Los períodos normales representaron el 63\% de los registros (figura 8). Estos resultados condicen con lo hallado por Scian y Donnari (1997) en diferentes regiones trigueras del sur de Argentina. Para ello, aplicaron el índice de Palmer y determinaron que para toda la región, las condiciones normales fueron las más frecuentes (40-50\%) mientras que los eventos severos y extremos de sequía alcanzaron los mayores porcentajes en el extremo sur. 
FIGURA 8

PORCENTAJE DE CADA CATEGORÍA DE SPEI, EN EL ÁREA

DE ESTUDIO (1970-2012)

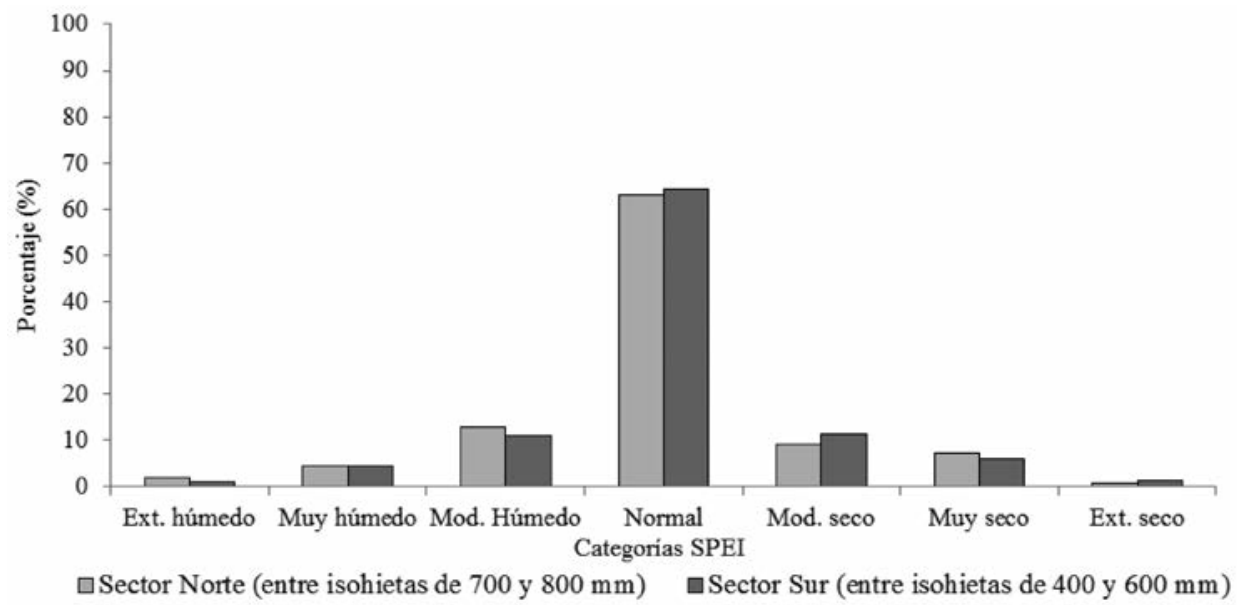

Fuente: elaborado sobre la base de los registros de SPEI (http: //sac.csic.es/spei/home.html).

Análisis de los parámetros agrícolas como indicadores de la alternancia de períodos normales, húmedos y secos

Tras el análisis de parámetros agrícolas (superficie sembrada, superficie cosechada y rendimientos) se obtuvo la evolución del cultivo de trigo en el área de estudio. El área cultivada promedio en el norte del territorio de estudio fue de 108000 ha mientras que en el sur de 172000 ha. El rendimiento promedio del cultivo de trigo para el período 2000/2012 fue de $1465 \mathrm{~kg} / \mathrm{ha}$ en el sur y de $2560 \mathrm{~kg} / \mathrm{ha}$ en el norte. Tomando en cuenta los registros de precipitación durante un período de 30 años, se obtuvo que el valor normal de precipitación. En la zona norte el valor normal de precipitación es de $780 \mathrm{~mm} / \mathrm{año}$ mientras que en el sur es de $377 \mathrm{~mm} / \mathrm{año}$.

En el sur del área de estudio, los rendimientos durante la campaña 2000/2001 estuvieron por encima del promedio. Se obtuvieron $1823 \mathrm{~kg} / \mathrm{ha}$ mientras que las precipitaciones también superaron al valor normal. Por el contrario, en el sector norte, los rendimientos fueron superiores al promedio y los registros de precipitaciones estuvieron cercanos al valor normal (figura 9). En esa campaña, la superficie sembrada, tanto en el norte como en el sur, superó al promedio y no se registraron pérdidas de superficie cosechada (figura 
9). En cambio, en la campaña 2001/2002 se obtuvieron diferencias apreciables entre el norte y el sur. Los rendimientos de trigo se mostraron superiores al promedio en el sur mientras que en el norte estuvieron cercanos al valor normal. En cuanto a las precipitaciones, éstas mostraron la misma tendencia.

\section{FIGURA 9}

\section{PRECIPITACIÓN ANUAL Y PARÁMETROS AGRÍCOLAS EN EL ÁREA DE ESTUDIO (PERÍODO 2000-2012)}

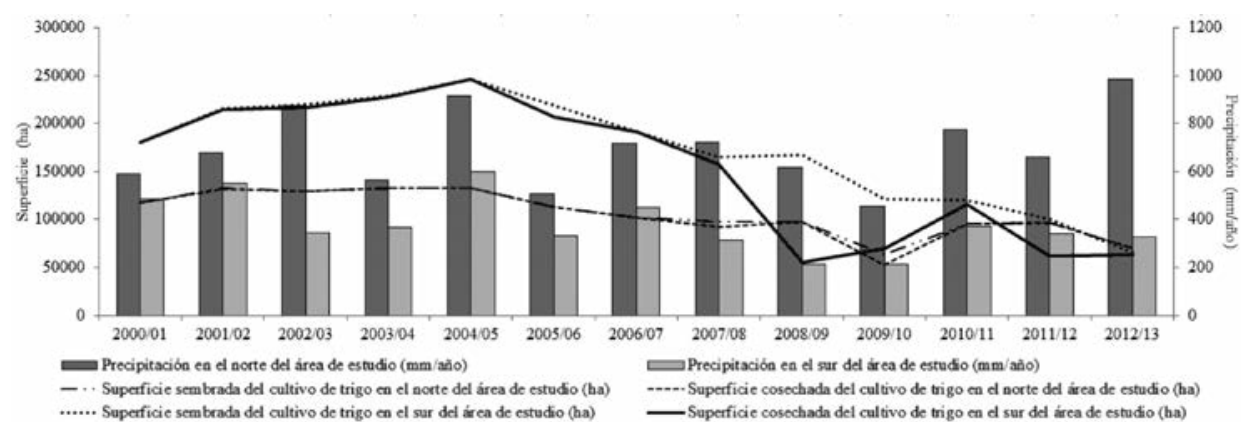

Fuente: elaborado sobre la base del SMN y MAGyP.

En la campaña 2002/2003, la ocupación del cultivo de trigo superó al valor promedio tanto en el norte como en el sur del área de estudio. Los rendimientos fueron cercanos y superiores al valor medio en el norte y en el sur, respectivamente. En el sur, los registros pluviométricos indicaron precipitaciones superiores a la normal (figura 9 y 10). La variabilidad en el régimen de precipitaciones de la RESAB está asociada a patrones de circulación general de la atmósfera (intensidad y posición de los anticiclones del Atlántico Sur y Pacífico Sur) (Zotelo, 2011) y a fenómenos de gran escala como el ENSO (Vargas et al., 1999).

En ambas zonas, la máxima superficie sembrada se obtuvo durante la campaña 2004/2005 y las precipitaciones fueron extremas en el sur del área de estudio $(599 \mathrm{~mm})$, al igual que el rendimiento (2235 kg/ha) (figura 9). En el norte se registraron $914 \mathrm{~mm}$ (por encima del valor normal) y el rendimiento también superó al promedio ( $2600 \mathrm{~kg} / \mathrm{ha}$ ). Durante el período analizado, se produjo el decrecimiento de la superficie sembrada a partir de la campaña 2006/2007 mientras que las precipitaciones tanto en el norte como en el sur del área de estudio, también disminuyeron respecto del promedio ( $377 \mathrm{~mm}$ en el sur y $780 \mathrm{~mm}$ en el norte) (figura 9). 
Durante la sequía extrema ocurrida en los años 2008 y 2009, en el sur del área de estudio, se obtuvieron pérdidas de superficie cosechada del 67\% (2008) y del 43\% (2009). En el norte, la sequía fue moderada y las pérdidas no superaron el 3\%. Las escasas precipitaciones durante estos años incidieron negativamente en los rendimientos (figura 10). En el sur, éstos fueron bajos, de 593 y $1667 \mathrm{~kg} / \mathrm{ha}$, y en el norte de 2000 y $1777 \mathrm{~kg} / \mathrm{ha}$, respectivamente. Los rendimientos de los principales cultivos disminuyeron considerablemente durante estos años en el oeste del área de estudio (D'Ambrosio et al., 2013). Según Coma (2010), en el área de estudio, se registraron pérdidas importantes en las cosechas de los años 2008 y 2009 en coincidencia con años de sequía. $\mathrm{Al}$ igual que los resultados hallados en la presente investigación y en coincidencia con lo estudiado por Magrin y Travasso (1997), el rendimiento de trigo en la región se mostró condicionado principalmente por las variaciones de la precipitación. En Bagdad, en los años 2008 y 2009 se produjo la segunda sequía de mayor impacto de la última década, provocando la disminución del $45 \%$ de la producción de trigo (Programa de Naciones Unidas para el Desarrollo, 2009).

Lo sucedido en el área de estudio en relación con la pérdida de superficie cosechada y los rendimientos en años secos concuerda con lo ocurrido en varias regiones semiáridas del mundo. En España, las sequías generaron importantes pérdidas en la agricultura de secano y los rendimientos de los cultivos se vieron muy reducidos durante los años secos (Austin et al., 1998; Iglesias et al., 2003). Las recurrentes pérdidas económicas agrícolas en el país y en el mundo permiten suponer aún una adaptación insuficiente a los eventos climáticos extremos (Wandel, 2006).

En la campaña 2010/2011, se hallaron similitudes en lo ocurrido entre el norte y el sur del área de estudio. En ambas zonas, las precipitaciones estuvieron cercanas al valor normal y los rendimientos $(3714 \mathrm{~kg} / \mathrm{ha}$ en el norte y $1727 \mathrm{~kg} / \mathrm{ha}$ en el sur) fueron superiores al promedio, mientras que durante la campaña siguiente (2011/2012) se obtuvieron diferencias. En el sur, la pérdida de superficie cosechada fue del $37 \%$ y las precipitaciones se encontraron por debajo del valor normal, como así también los rendimientos (1013 kg/ha) (figura 10). En el norte, no se obtuvieron pérdidas de superficie cosechada y las precipitaciones se acercaron al valor normal, mientras que los rendimientos fueron de $3190 \mathrm{~kg} / \mathrm{ha}$ (superiores al promedio).

En la campaña 2012/2013 también se encontraron diferencias en el área de estudio. Las precipitaciones en el norte fueron las máximas de todo el período analizado $(985 \mathrm{~mm})$ como así también los rendimientos (3986 kg/ha). La zona sur, se caracterizó por precipitaciones por debajo del valor normal (326

Estudios Geográficos, Vol. LXXVIII, 282, pp. 7-29, enero-junio 2017

ISSN: 0014-1496, eISSN: 1988-8546, doi: 10.3989/estgeogr.201701 
$\mathrm{mm}$ ) como así también los rendimientos (1300 kg/ha) (figura 10). No se registraron pérdidas de superficie cosechada en el norte mientras que en el sur se aproximaron al 4\%. De manera general, observando la evolución en los rendimientos del cultivo de trigo, se puede inferir la tendencia de la precipitación en el área de estudio. En la mayoría de los casos, los máximos rendimientos estuvieron asociados a años con precipitaciones anuales por encima del valor normal mientras que los rendimientos disminuyeron considerablemente en años secos. Esto coincide con lo hallado por D'Ambrosio et al. (2011), durante la sequía del año 2008-2009 en el área de estudio. Estos afirmaron que los rendimientos de los principales cultivos disminuyeron considerablemente durante este evento. En relación con ello, Capa et al. (2012) mostraron que la variabilidad climática y los cambios en la frecuencia de los eventos extremos tienen un impacto directo sobre los rendimientos de los cultivos. Las pérdidas económicas en las sequías suelen llegar a niveles que afectan severamente la continuidad de las explotaciones agropecuarias regionales (Carbonell, 2010).

FIGURA 10

PRECIPITACIÓN ANUAL Y RENDIMIENTO DE TRIGO EN EL ÁREA DE ESTUDIO

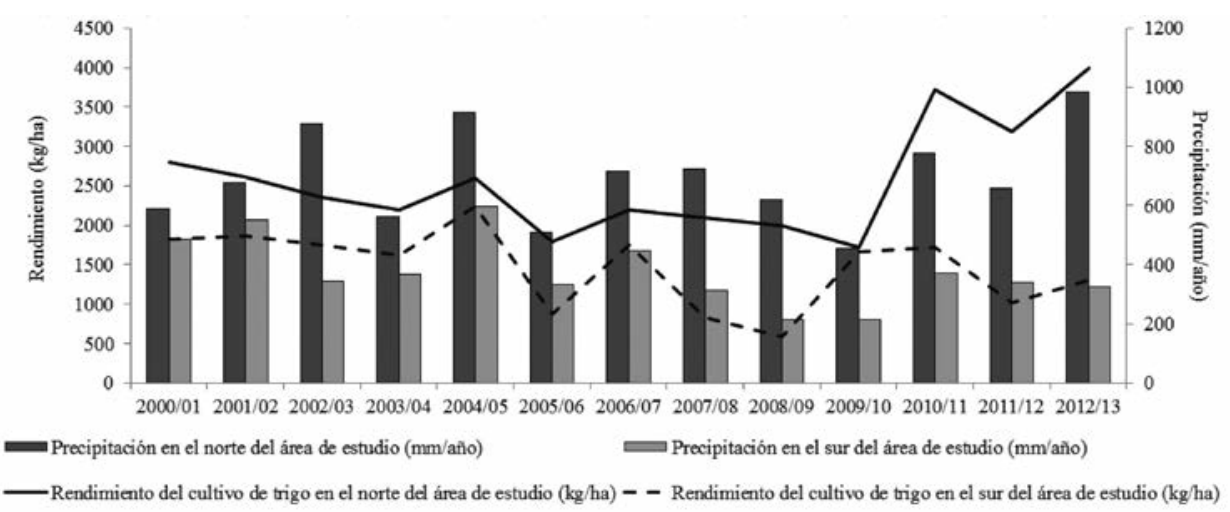

Fuente: elaborado sobre la base de registro del SMN y MAGyP.

\section{Rendimientos de trigo como indicadores de las variaciones del SPEI}

Los años 2001 y 2008 fueron definidos como húmedos y secos, respectivamente. En 2001 (figura 11a), los rendimientos de trigo en la mayor parte de la región fueron superiores al promedio. Hacia el norte los rendimientos oscilaron entre 2000 y $2600 \mathrm{~kg} / \mathrm{ha}$ y los valores de SPEI entre 1.5 y 1.99 (muy hú- 
medo). En el sector sur, los rendimientos también estuvieron por encima del promedio aunque fueron menores que en el sector norte. Se obtuvieron desde 1800 a $2000 \mathrm{~kg} / \mathrm{ha}$ mientras que los valores de SPEI fueron categorizados como de moderada humedad (figura 1la). En 2008 (figura 1lb), el rendimiento de trigo estuvo por debajo del promedio en la totalidad del área de estudio. Hacia el norte la sequía fue moderada y los rendimientos fueron más altos que en el sur. Hacia el sur, donde las condiciones fueron muy secas y extremadamente secas (SPEI entre -1.5 y -1.99 y -2.0 o menos) los rendimientos oscilaron entre 500 y $1000 \mathrm{~kg} / \mathrm{ha}$ (figura 11b).

\section{FIGURA 11}

\section{RELACIÓN ENTRE LAS CATEGORÍAS EXTREMAS DEL SPEI HÚMEDAS (A) Y} SECAS (B) Y LOS RENDIMIENTOS DE TRIGO
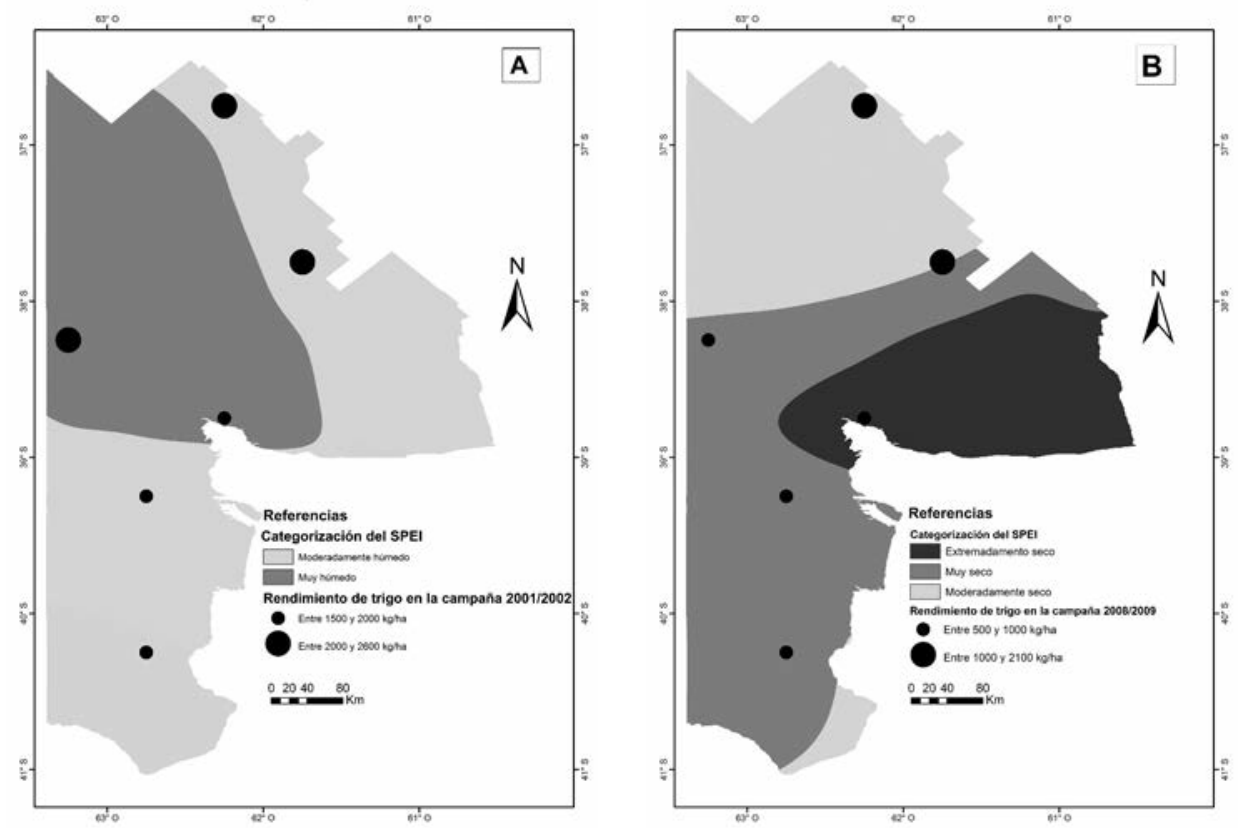

Fuente: elaborado sobre la base de los registros de SPEI (http: //sac.csic.es/spei/home.html). 


\section{CONCLUSIÓN}

La aplicación del SPEI es novedosa y apropiada para el análisis de períodos secos, húmedos y normales en el área de estudio. Mediante esta metodología se ha obtenido la tendencia de la precipitación, destacando como principal característica la alternancia de eventos secos y húmedos, de diferente intensidad y duración.

Durante el período 1970-2012, los ciclos normales fueron los más frecuentes. Sin embargo, hubo una desigual proporción de fases secas y húmedas entre el norte y el sur del área de estudio. En el norte predominaron los períodos húmedos y en menor medida los secos. En el sur, ambas categorías obtuvieron porcentajes similares. Por lo tanto, durante los 42 años estudiados no se observó un aumento significativo en la frecuencia de períodos húmedos y secos.

Los eventos húmedos más extensos ocurrieron durante la década de 19701980 mientras que los secos más prolongados se presentaron a partir de 2005 hasta el año 2009 en el norte y desde el 2008 hasta 2011 en el sur de la región estudiada. Como ejemplo de años secos se destacaron los años 2008 y 2009 ocasionando consecuencias negativas para la actividad agrícola de la región.

El área de estudio forma parte de una de las áreas trigueras más importantes del país. El rendimiento del mencionado cultivo así como la superficie cosechada con respecto a la sembrada, disminuyeron de forma notoria durante la sequía del 2008/2009. En toda la región, la mayor pérdida de superficie cosechada ocurrió durante la misma campaña temporal; la zona sur fue la más afectada. Por su parte, el año 2001 fue húmedo tanto en el norte como en el sur del área de estudio y el rendimiento durante dicho año fue uno de los más altos del período estudiado. A diferencia de los años secos, los rendimientos del cultivo de trigo se mostraron por encima del promedio durante los períodos húmedos. De esta manera, estos se manifestaron como un indicador de los períodos secos y húmedos. Sin embargo, existen otros factores que limitan el rendimiento de trigo y que deben ser estudiados más profundamente. Entre ellos se destacan: la disponibilidad de nitrógeno y fósforo, las propiedades edáficas, fecha de siembra, entre otros. No obstante, todas las investigaciones indican que la variable de mayor impacto sobre el rendimiento de trigo es la precipitación.

Una de las principales consecuencias de la alternancia de ciclos secos y húmedos que caracteriza al área de estudio es la desestabilización de los rendimientos de las actividades productivas y las grandes dificultades para cualquier tipo de planificación agropecuaria que se proyecte. Ante esta situación, algunas medidas de adaptación podrían involucrar la diversificación de 
los cultivos, incorporando nuevas especies que se adapten mejor a la periodicidad de estos eventos, facilitar a los productores agropecuarios una mayor accesibilidad a la información de pronósticos de tiempo confiables y eficaces para la toma de decisiones acertadas, mejoramiento en la eficiencia del uso del agua, entre otras. El presente estudio ha servido para cuantificar y conocer mejor la periodicidad y frecuencia de este tipo de sucesos y su influencia sobre el principal cultivo de la región.

\section{Agradecimientos}

La presente investigación fue realizada mediante subsidios otorgados por el Consejo Nacional de Investigaciones Científicas y Técnicas (CONICET) y la Universidad Nacional del Sur (UNS). Las autoras agradecen al Servicio Meteorológico Nacional (SMN) y el Instituto Nacional de Tecnología Agropecuaria (INTA) por los datos cedidos.

\section{BIBLIOGRAFÍA}

Alvarez R. y Lavado, R. S. (1998): "Climate, organic matter and clay content relationships in the Pampa and Chaco soils, Argentine", Geoderma, 83, pp. 127-141.

Askew, A. y Rooda, J. (1995): "Addressing arid zone problems, L'hydrologie tropicale: séoscience outil pour le développement", en Actes de la conférence de Paris, París, AHS Publ., p. 238.

Austin, R., Cantero-Martínez, C., Arrúe, J. L., Playán, E. y Cano-Marcellán, P. (1998): "Yield-rainfall relationships in cereal cropping systems in the Ebro River valley of Spain", European Journal of Agronomy, 8, pp. 239-248.

Bannayana, M., Sanjania, S., Alizadeha, A., Sadeghi Lotfabadia, S. y Mohamadianb, A. (2010): "Association between climate indices, aridity index, and rainfed crop yield in northeast of Iran", Field Crops Research, 118/2, pp. 105-104.

Bohn, V. Y., Piccolo, M. C. y Perrillo, G. M. E. (2011): "Análisis de los períodos secos y húmedos en el sudoeste de la provincia de Buenos Aires (Argentina)", Climatología, 11, pp. 31-43.

Brown, A., Martínez Ortiz, U., Acerbi, M. y Corcuera, J. (2006): La Situación Ambiental Argentina 2005, Buenos Aires, Fundación Vida Silvestre Argentina, 587 pp.

Campo, A., Bagnulo, C. y Pacheco, R. (2012): "Cambios en el régimen pluviométrico y sus efectos sobre la producción agropecuaria en el partido de Puan", Revista Electrónica Geografía Austral, 1, pp. 1-11.

Campo, A., Capelli, A. y Diez, P. (2004): El clima del Sudoeste Bonaerense, Bahía Blanca, EdiUns, 99 pp. 
Capa, M., Rodríguez Fonseca, B. y Ruiz Ramos, M. (2007): "Impactos de la variabilidad climática en los cultivos de verano en regadío mediterráneos", en VIII Congreso Internacional de Cambio Climático, Salamanca, Spanish Association of Climatology Publications, pp. 747-755.

Carbone, M. E., Scian, B. y Piccolo, M. C. (2008): "Agricultural drought in the Claromeco river basin, Buenos Aires province, Argentina”, Revista de Climatología, 8, pp 1-13.

Carbonell, C. (2010): "Relación de las precipitaciones primaverales sobre los rendimientos de trigo en la región de influencia de Bahía Blanca en el sudoeste bonaerense semiárido", en XIII Reunión Argentina y VI Latinoamericana de Agrometeorología (RALDA), Bahía Blanca, Asociación Argentina de Agrometerología, pp. 1-6.

Castellarín, J., Pedrol, H., Salvagiotti, F. y Rosso, O. (1999): "Evaluación de cultivares de trigo PAN en la localidad de Oliveros Campaña 1998/1999", Trigo para mejorar la producción 9 Campaña 1998/99, Rosario, INTA EEAA Oliveros, pp. 37-40.

Coma, C. (2010): "Trigo en el área de la EEA INTA BORDENAVE", en Informe Técnico, provincia de Buenos Aires, Buenos Aires, Instituto Nacional de tecnología Agropecuaria, pp. 1-19.

Coronel, A., Isoardi, C., Mainardi, L., Mascotti, G. y Tamantini, G. (2004): "Características sinópticas de la sequía que afectó a la localidad de Zavalla de agosto a octubre de 1998 y su relación con el rendimiento del trigo", Revista de Investigaciones de la Facultad de Ciencias Agrarias, 5, pp. 31-44.

D’Ambrosio, G. (2011): La sequía 2008-2009 en el Oeste de la región pampeana, tesis de licenciatura en Geografía, Universidad Nacional del Sur, Departamento de Geografía y Turismo, Argentina, 82 pp.

D’Ambrosio, G., Bohn, V. Y. y Piccolo, M. C. (2013): "Evaluación de la sequía 20082009 en el oeste de la Región Pampeana", Cuadernos Geográficos, 52/1, pp. 29-45.

Diaz, R. y Mormeneo, I. (2002): "Zonificación del clima en la región pampeana mediante análisis de conglomerado por consenso", Agrometeorología, 2, pp. 125-131.

Diovisalvi, N., Bohn, V. Y., Piccolo, M. C., Perillo, G. M. E., Baigún, C. y Zagarese, H. E. (2015): "Shallow lakes from the Central Plains of Argentina: an overview and worldwide comparative analysis of their basic limnological features", Hydrobiologia, 752, pp.5-20.

Fernandez, B. y Vergara, A. (1998): "Risk of scarcity of monthly precipitation and streamflows in 490 semiarid regions", Hydrological Sciences Journal, 5, pp. 59-77.

García Marín, R. (2006): "Evolución y tendencias de la precipitación estacional en la cuenca del Guadalentín (Murcia-Almería): posibles efectos en la práctica agrícola de secano", Nimbus: Revista de climatología, meteorología y paisaje, 17, pp. 43-65.

Gómez, C. V. y Pérez, M. E. (2011): "Los períodos secos en la provincia del chaco entre los años 1957 y 2005”, Revista Geográfica Digital, IGUNNE, Facultad de Humanidades, 15, pp. 1-19.

Grondona, M. O., Magrin, G. O., Travasso, M. I., Moschin, R. C., Rodríguez, G. R., Messina, C., Boullón, D. R. y Podestá, G. (2007): Impacto del fenómeno El Niño so- 
bre la producción de trigo y maíz en la región pampeana argentina, Castelar, Argentina, Instituto de Tecnología Agropecuaria.

Hall, A., Rebella, C. M., Ghersa, C. y Culot, J. P. (1992): "Field crops systems of the Pampas", Ecosystems of the World, 18, pp. 1-15.

Horridge, M., Madden, J. y Wittwer, G. (2005): "The impact of the 2002-2003 drought on Australia", Journal of Policy Modeling, 27, pp. 285-308.

Hurtado, R. y Berri, G. (2001): "Relación entre los rendimientos trigueros y el fenómeno del ENSO en la región pampeana en el periodo 1970-1997", X Congresso Brasileiro de Meteorologia, Brasilia, Brasil, inédita, pp. 1-17.

Iglesias, E., Garrido, A. y Gómez-Ramos, A. (2003): "Evaluation of drought management in irrigated areas", Agricultural Economics, 29, pp. 211-229.

Iriondo, M. H. (1989): "Quaternary lakes of Argentina", Palaeogeography, Palaeoclimatology, Palaeoecology, 70, pp. 81-88.

López Bermúdez, F. y Sánchez Fuster, M. C. (1997): "Las sequías y su impacto en el riesgo de desertificación de la cuenca del Segura Apuntes para la gestión y sustentabilidad del agua", Áreas, Revista Internacional de Ciencias Sociales, 17, pp. 155168.

Magrin, G. y Travasso, M. (1997): "Potencial de producción del cultivo de trigo en la Región Pampeana argentina”, en Actas Reunión de Planificación Estratégica de Trigo, Mar del Plata, Buenos Aires, Argentina, INTA-CIMMYT, pp. 34-35.

Mc Kee, T., Doesken, N. y Kleist, J. (1995): "Drought monitoring with multiple time scales", en Ninth Conference on Applied Climatology, Boston, American Meteorological Society, pp, 223-236.

Piccolo, M., Capelli, A. y Campo, A. (2002): "La sequía de 1995 en el sur de la región pampeana argentina", en Desastres Naturales en América Latina, Buenos Aires, Fondo de Cultura Económica, pp. 189-206.

Ponzio, N. y Adam, S. (2013): "El trigo pilar del desarrollo local", http://www.diarioeltiempo.com.ar/archivo/2013/el-trigo-pilar-del-desarrollo-local.html (fecha de consulta: 15 de marzo de 2015).

Programa de Naciones Unidas para el Desarrollo (2009): "Iraq: combatir los efectos de la sequía debido al cambio climático", http://www.undp.org/content/undp/es/ home/presscenter/articles/2009/12/16/undp-helps-drought-stricken-iraq-combateffects-of-climate-change.html (fecha de consulta: 22 de marzo de 2015).

Rivera, J. A. (2014): Aspectos climatológicos de las sequías meteorológicas en el sur de Sudamérica. Análisis regional y proyecciones futuras, tesis doctoral, Universidad de Buenos Aires, Facultad de Ciencias Exactas, 352 pp.

Satorre, E. H. y Slafer, G. A. (eds.) (1999): Wheat production systems of the Pampas. Wheat, Ecology and Physiology of Yield Determination, New York, Haworth press, 348 pp.

Scian, B. (2002): "Variabilidad de las condiciones hídricas en la región semiárida pampeana Argentina", Geoacta, 27, pp. 30-52.

Sebalt, W. (2002): Relación agricultura-ganadería en la zona semiárida de la región pam-

Estudios Geográficos, Vol. LXXVIII, 282, pp. 7-29, enero-junio 2017

ISSN: 0014-1496, eISSN: 1988-8546, doi: 10.3989/estgeogr.201701 
peana, área comprendida por los partidos de Villarino, Patagones y sur de Puan como alternativas para optimizar la empresa agropecuaria, tesis de magister, Universidad Nacional del Sur, Departamento de Ciencias de la Administración, Argentina, 188 pp.

Vargas, W. M., Penalba, O. y Minetti, J. (1999): "Las precipitaciones mensuales de la Argentina y el ENOS. Un enfoque hacia el problema de la decisión", Meteorológica, 24, pp. 3-18.

Vicente-Serrano, S. M., Beguería, S. y Lopez-Moreno, J. I. (2010): “A Multiscalar Drought Index Sensitive to Global Warming: The Standardized Precipitation Evapotranspiration, Index", Journal of Climate, 23/7, pp. 1696-1718.

Viglizzo, E. F., Frank, F. C., Carreño, L., Jobbágy, E., Pereyra, H., Clatt, J., Pincén, D. y Ricard, F. (2011): "Ecological and environmental footprint of 50 years of agricultural expansion in Argentina”, Global Change Biology, 17, pp. 959-973.

Wandel, J. (2006): "Adaptation, adaptive capacity and vulnerability", Global Environmental Change, 16, pp. 282-292.

Zotelo, C. (2011): "Variabilidad climática y ciclos naturales", en Jornadas sobre Evolución y Futuro del Desarrollo de Producciones Agrícola, Bahía Blanca, Academia Nacional de Agronomía y Veterinaria. Trabajos del tomo LXV, pp. 374-391.

Fecha de recepción: 15 de junio de 2015.

Fecha de aceptación: 30 de diciembre de 2015. 